\title{
Friction Compensation in the Upsetting of Cylindrical Test Specimens
}

\author{
Christiansen, Peter; Martins, P. A. F.; Bay, Niels Oluf
}

Published in:

Experimental Mechanics

Link to article, DOI:

$10.1007 / \mathrm{s} 11340-016-0164-z$

Publication date:

2016

Document Version

Peer reviewed version

Link back to DTU Orbit

Citation $(A P A)$ :

Christiansen, P., Martins, P. A. F., \& Bay, N. O. (2016). Friction Compensation in the Upsetting of Cylindrical Test Specimens. Experimental Mechanics, 56(7), 1271-1279. https://doi.org/10.1007/s11340-016-0164-z

\section{General rights}

Copyright and moral rights for the publications made accessible in the public portal are retained by the authors and/or other copyright owners and it is a condition of accessing publications that users recognise and abide by the legal requirements associated with these rights.

- Users may download and print one copy of any publication from the public portal for the purpose of private study or research.

- You may not further distribute the material or use it for any profit-making activity or commercial gain

- You may freely distribute the URL identifying the publication in the public portal

If you believe that this document breaches copyright please contact us providing details, and we will remove access to the work immediately and investigate your claim. 
FRICTION COMPENSATION IN THE UPSETTING OF

\title{
CYLINDRICAL TEST SPECIMENS
}

\author{
P. Christiansen ${ }^{1 *}$, P.A.F. Martins ${ }^{2}$, N. Bay ${ }^{1}$ \\ ${ }^{1}$ Department of Mechanical Engineering, Technical University of Denmark, DTU - Building 425, \\ DK-2800 Kgs. Lyngby, Denmark \\ ${ }^{2}$ Instituto Superior Tecnico, Universidade de Lisboa, Av. Rovisco Pais, 1049-001 Lisboa, Portugal
}

*Corresponding author: E-mail: petc@mek.dtu.dk, Tlf: +45 45254753 


\begin{abstract}
This manuscript presents a combined numerical and experimental methodology for determining the stress-strain curve of metallic materials from the measurements of force and displacement obtained in the axial compression of cylindrical test specimens with friction between the specimens and the platens. The methodology is based on minimizing the error between the average surface pressure obtained from the experimental measurements of the force and displacement and that obtained from the slab method of analysis of metal plasticity. Three different friction models based on Coulomb friction, the constant friction model or combined friction models are utilized.

Experimental results obtained from cylindrical and Rastegaev test specimens with different lubricants combined with the experimental determination of friction by means of ring compression tests allows compensating the effect of friction in the determination of the material flow curve. Comparison with the flow curves determined without friction compensation shows the viability of the proposed methodology.

The proposed methodology is a simple and effective alternative to other solutions available in the literature and the pseudo-code supplied in the Appendix is provided for those readers interested in utilizing the associated numerical algorithm for determining the stress-strain curves of metallic materials.
\end{abstract}

\title{
Keywords
}

Compression test, friction compensation, stress-strain curve 


\section{Introduction}

The compression test consists of the upsetting of a cylindrical test specimen between flat parallel platens and is a widely utilized method for obtaining the flow curve of metallic materials. From a metal forming point of view, the flow curve is one of the most important material data for modelling the mechanical behavior of metals because it is utilized to describe strain hardening during plastic deformation, to set-up the nonlinear constitutive equations of plasticity, to estimate the forces and pressures applied on workpiece and tools and to determine the process operating conditions, among other scientific and practical engineering utilizations.

If it were possible to completely eliminate friction from the upset compression tests, the flow curve could easily be determined from the experimental values of force and displacement because the specimens would deform homogeneously and the diameter $d$ would be uniformly constant along the height $h$.

However, there are several reasons for not achieving homogeneous plastic deformation conditions in daily practice. First, the overall experimental procedure is strongly dependent on the quality of the lubricants applied on both specimens and platens. Second, because it is not possible to ensure frictionless conditions, even with the most efficient lubricants, there will always be signs of 'barrelling' and, therefore, there will always be residual friction associated with the upset compression test. This amount of residual friction needs to be identified and subsequently eliminated from the flow curve. And third, even in case of using specially designed test specimens such as that proposed by Rastegaev [1], in which a lubricant reservoir is included on the top and bottom surfaces in order to encapsulate the lubricant and prevent direct metal to metal contact on most of the interface between specimen and platens, there will always be signs of friction, namely when the pressurized lubricant in the reservoir escapes outward.

The alternative of using thin sheets of teflon for eliminating friction is also limited by tearing of the sheets of teflon and subsequent metal to metal contact between specimen and platens. Moreover, if 
the aim is to perform upset compression tests in warm and hot forming regimes, it may be difficult to use either teflon sheets or conventional lubricants due to the risk of fire. Therefore, it is of importance to be able to correct the flow curve that is directly obtained from the upset compression tests with friction.

Cook and Larke [2] were among the first researchers to remove the frictional work done in upset compression tests. They proposed a methodology in which four specimens with different initial $h / d$ ratios (2, 1, 1/2 and 1/3) are compressed under constant lubrication conditions and their results extrapolated in a systematic way to give the desired flow curve for a specimen of infinite $h / d$ ratio. The justification behind Cook and Larke's methodology is based on the fact that as the height $h$ of the compression test specimens increases, the influence of the 'conical dead zones' that form in each end of the specimen, below the compression platens, decreases so that in the limit (when the specimen has an infinite height $h$ ) this influence is negligible and plastic deformation may be considered homogeneous. The reason why the initial ratios $h / d$ are limited to $3: 1$ is to prevent specimens from buckling instead of being ideally compressed in its height direction.

In 1963 Alexander and Brewer [3] revisited the utilization of Cook and Larke's method and proposed a modification based on the utilization of equal values of force instead of equal values of reduction in height, as it was initially proposed by Cook and Larke [2]. If, in addition to this, the test is performed with increments of force instead of continuous loading, as it was originally carried out by Watts and Ford [4] in case of the plane strain compression of strips between parallel platens, it is possible to remove the specimens from the testing machine at regular intervals to renew lubrication and significantly reduce the size of friction effects on the experimental force. Removal of the specimens from the testing machine also allows the height to be measured without having to account for the elastic deformation of the tools.

In 1977 Woodward [5] changed the extrapolating procedure associated with the different variants of Cook and Larke's method [2] into an interpolating procedure based on the utilization of a corrective 
mathematical function obtained from Avitzur's [6] upper bound solution for the compression of a cylinder between flat parallel platens. The method makes use of two upset compression tests instead of the four upset compression tests required by Cook and Larke's method [2] but requires the specimens and the platens to be very well lubricated. In fact, Woodward [5] suggested the teflon sheets to be renewed at regular intervals during compression so that uniform compression is achieved and the effect of friction on the measured compression load is therefore initially small.

Osakada et al. [7] obtained stress-strain curves of metals by means of an inverse analysis using a rigid-perfectly plastic finite element method. Various compensations due to deformation hardening and temperature increase had to be performed and eventually comparisons with upsetting tests using thin teflon sheets was performed. The method of applying FEM analysis requires availability of a FEM program by the laboratory or workshop. This may limit the applicability of the method.

In 2002 Han [8] modified Woodward's method [5] by assuming the flow stress for a given material to be independent from the geometry of the specimen, and thereby were able to set up an objective function dependent on friction stress. The coefficient of friction and the flow stress are determined by means of an inverse computational procedure that makes use of the objective function built upon the slab method solution for the compression of a cylinder between flat parallel platens [9]. Xinbo et al. [10] proposed an alternative procedure in which an objective function and subsequent optimization procedure are built upon the differences between the experimental and finite element estimates of the total compression force.

In addition to the above mentioned procedures for determining the flow curve directly from the force-displacement evolution obtained in the experiments and indirectly from the utilization of mathematical or numerical procedures that eliminate the effects of friction from the compression force, there are other approaches focused on the characterization of the friction directly from the barreled surface of the upset compression test specimens. Tan et al. [11], for example, developed a procedure to determine friction by applying the relative shrinking ratio of the original end surface of 
the test specimens as a calibration parameter. Once friction is determined, it is easy to determine the flow curve of the material from the experimental measurements of force and displacement.

From what was mentioned above, it may be concluded that the problem of determining the flow curve directly from upset compression tests with friction requires specific methods and procedures that are different from those included in classical tribology publications dealing with friction, lubrication and surface/interface kinematics (Wilson [12]).

Under these circumstances, this paper proposes a simple and effective methodology to determine the stress-strain curve from the experimental measurements of force and displacement in testing conditions where friction between the cylindrical specimen and the platens would cause barreling of the outer surface. In contrast to other optimization based methods, the proposed approach accounts for the changes in friction arising from the differences in pressure at the center and at the edges of the test specimens by making use of objective functions that are built upon the slab method of analysis using Coulomb friction or the constant friction model. Combined friction models (not to be confused with mixed lubrication) where Coulomb friction is more appropriate for modelling the low pressures found at the edge of the specimens and constant friction is more adequate for modelling the high pressures found at the center of the specimens are taken into account. The stress-strain curves resulting from the new proposed method are fitted by well-known strain hardening material models.

The experimental data giving support to the presentation was obtained by means of upset compression tests performed with Rastegaev [1] test specimens and cylindrical test specimens lubricated either with grease or thin sheets of teflon on top and bottom. Friction was calibrated independently by means of ring compression tests and the corresponding values were utilized to assess the predicted values of friction that were determined from the new proposed method.

A pseudo-source code of the MATLAB computer program that was utilized in the investigation is provided in the Appendix for the readers interested in applying the new proposed methodology in daily practice. 


\section{Theoretical background}

\section{Objective function}

The proposed methodology provides a mathematical approximation of the stress-strain curve by means of the strain hardening material models that are listed in Table 1 [13-16] from the experimental evolution of the force with displacement in upset compression tests performed with friction. The approach is developed for cold forming, where rate effects are negligible and the flow stress $\sigma_{0}$ is commonly assumed to be a function only of the effective plastic strain $\bar{\varepsilon}$ (hereafter designated as 'the effective strain'). Strain rate and/or temperature effects could also be included in the algorithm but are not considered in the present article.

$$
\begin{array}{ll}
\text { Table } 1 \text { Strain hardening material models } \\
\text { Hollomon } & \sigma_{o}=C \bar{\varepsilon}^{n} \\
\text { Swift } & \sigma_{o}=C(B+\bar{\varepsilon})^{n} \\
\text { Ludwik } & \sigma_{o}=K \bar{\varepsilon}^{n}+\sigma_{y} \\
\text { Voce } & \sigma_{o}=a+(b-a)\{1-\exp (-c \bar{\varepsilon})\}
\end{array}
$$

The effective strain under frictionless compression conditions is given by $\bar{\varepsilon}=\ln \left(H_{o} / H_{1}\right)$, where $H_{o}$ is the initial and $H_{1}$ is the final height of the cylinder specimens. The corresponding flow stress $\sigma_{o}$ is computed by:

$$
\sigma_{o}=\bar{p}
$$

where $\bar{p}$ is the average surface pressure that coincides with the pointwise distribution of pressure $p$ in the absence of friction. 
The objective function for determining the flow curve is given by $f\left(M, \tau_{s}\right)=\left|\bar{p}_{\text {exp }}-\bar{p}\right|$ where $M$ denotes the independent parameters of the strain hardening material model, $\tau_{s}$ is frictional shear stress at the contact interface between the specimen and the platens, $\bar{p}_{\text {exp }}$ is the experimental average surface pressure and $\bar{p}$ is the corresponding estimate of average pressure obtained from the slab method solution for the compression of a cylindrical test specimen with diameter $D$ and height $H$ between flat parallel platens [8]. Two models are taken into consideration for modelling the frictional effects at the contact interface between the specimen and the platens: the Coulomb friction model given by $\tau_{s}=\mu p$, where $\mu$ is the friction coefficient and $p$ is the normal pressure,

$\bar{p}=2 \sigma_{o}\left(\frac{H}{\mu D}\right)^{2}\left[\exp \left(\frac{\mu D}{H}\right)-\frac{\mu D}{H}-1\right]$

and the constant friction model $\tau_{s}=m k$, where $m$ is the friction factor $(0 \leq m \leq 1)$ and $k$ is the yield stress of the material in pure shear,

$\bar{p}=\sigma_{o}\left(1+\frac{m D}{3 \sqrt{3} H}\right)$

Similarly to the slab method of analysis, the mathematical approximation of the stress-strain curve by means of the new proposed procedure is based on the following three assumptions; (i) the principal axes are in the directions of the applied loads, (ii) the effects of friction do not change the directions of the principal axes, and (iii) plane sections remain plane during compression.

In contrast to other approaches available in literature, the proposed methodology is capable of combining the two above estimates of average pressure (eq. (2) and eq. (3)) in order to account for the differences in lubrication that are found between the center and the edge of the cylindrical test specimens. These differences are due to the fact that during compression lubricant runs out of the 
edges and the barreled surface folds up onto the compression platens giving rise to dry metal-tometal contact. As a result of this, the frictional shear stresses are higher at the edges than in the central region of the specimens where lubricant becomes trapped. The same situation applies with the utilization of thin sheets of teflon, which are cut out by the edges of the specimens during compression.

In case of combining the two above mentioned friction models, it is considered that the constant friction model is the most adequate for the central region of the specimen and the Coulomb friction model for the outer region $\left(0 \leq r_{g} \leq R, R=D / 2\right)$, where $r_{g}$ is the transition radius between the two friction models:

$\bar{p}=\sigma_{o}\left(\frac{m}{\sqrt{3}}\left[\frac{1}{\mu}\left(\frac{r_{g}}{R}\right)^{2}+\frac{2 r_{g}^{3}}{3 H R^{2}}\right]+\frac{1}{2 \mu^{2}}\left(\frac{H}{R}\right)^{2}\left[\left(1+\frac{2 \mu r_{g}}{H}\right) \exp \left\{\frac{2 \mu\left(R-r_{g}\right)}{H}\right\}-\frac{2 \mu R}{H}-1\right]\right)$

In case $r_{g}=0$ only the Coulomb friction model is utilized and in case of $r_{g}=R$ and $\mu=m / \sqrt{3}$ only the constant friction model is utilized. For numerical implementation purposes, combination of the two friction models required the utilization of a modified version of eq. (4), in which the radius $r_{g}$ is expressed by means of a fixed fraction $x$ of the outer radius $R\left(r_{g}=x R, 0 \leq x \leq 1\right)$ :

$\bar{p}=\sigma_{o}\left(\frac{m}{\sqrt{3}}\left[\frac{1}{\mu} x^{2}+\frac{2 x^{3} R}{3 H}\right]+\frac{1}{2 \mu^{2}}\left(\frac{H}{R}\right)^{2}\left[\left(1+\frac{2 \mu x R}{H}\right) \exp \left\{\frac{2 \mu(1-x) R}{H}\right\}-\frac{2 \mu R}{H}-1\right]\right)$

Eq. (5) will be used later in the article for the comparison between experimental and computed compression forces. 


\section{Numerical implementation}

The proposed method for determining the stress-strain curve initiates with a first guess of the stressstrain curve obtained from the experimental evolution of the force with displacement retrieved from the compression test specimens. This first guess assumes that the compression test specimens experience homogenous plastic deformation.

The stress strain curve of the material is then fitted by one of the strain hardening material models listed in Table 1 and the corresponding independent parameters (for example, $C$ and $n$ in case of the Hollomon $\sigma_{o}=C \bar{\varepsilon}^{n}$ strain hardening material model) are subsequently determined by means of a numerical procedure aimed at minimizing the objective function $f\left(M, \tau_{s}, x\right)=\left|\bar{p}_{\text {exp }}-\bar{p}\right|$. The minimum is considered reached when the change in the solution becomes sufficiently small

$\frac{\Delta\left|\bar{p}_{\text {exp }}-\bar{p}\right|}{\left|\bar{p}_{\text {exp }}\right|}<\delta$, where $\Delta\left|\bar{p}_{\text {exp }}-\bar{p}\right|$ is the difference between the previously best and current best residual.

The simplest algorithm that can be utilized to determine the independent parameters of the strain hardening material model and of the friction coefficient (and/or friction factor) brackets the root of $f\left(M, \tau_{s}, x\right)$ within a search interval $\left[M_{a}, \tau_{s a}, x_{a} ; M_{b}, \tau_{s b}, x_{b}\right]$. Knowledge of the typical order of magnitude of the independent parameters and friction values helps limiting the search interval to values in ranges that are meaningful from a plastic deformation point of view. Say, for example, $0 \leq n \leq 0.5,0 \leq m \leq 1$ and $0 \leq \mu \leq 1 / \sqrt{3}$.

A pseudo-code, written in MATLAB, of the proposed methodology regarding how to perform friction correction in upset compression tests, is provided in the Appendix. Further information on the associate numerical algorithm is available in standard textbooks [17]. 


\section{Experimental procedure}

\section{Upsetting of cylindrical and Rastegaev compression test specimens}

The investigation was performed on Aluminium AL2S (99.7\% Al, 0.2\% Fe, 0.1\% Si) supplied in the form of $30 \mathrm{~mm}$ diameter rods. The cylindrical and Rastegaev test specimens were machined in accordance to the geometries shown in Fig. 1 and the experiments were carried out with the material in the 'as-supplied' condition. The specimens where measured before deformation using a Vernier caliper.
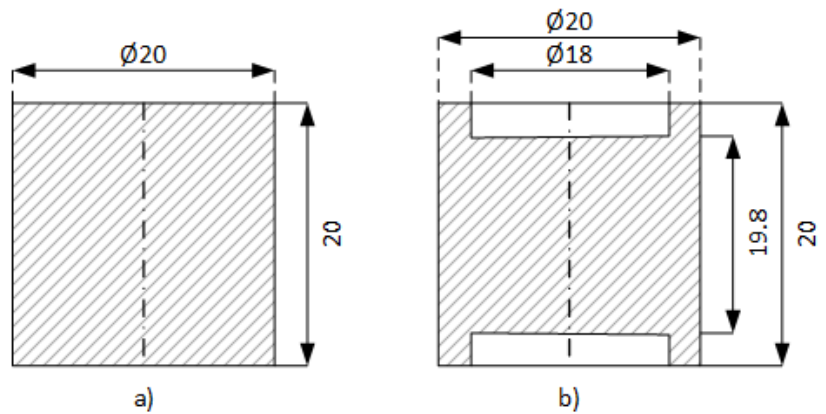

b)

Fig. 1 Geometry of the a) Cylindrical and b) Rastegaev compression test specimens. Figures not drawn to scale

The upset compression tests were performed in a hydraulic press with constant moving cross-head speed and the force-displacement evolution was recorded on PC for each test. The compression platens were cleaned with ethanol before each experiment. The cylinder test specimens were lubricated with Molykote DX paste or teflon sheets on the top and bottom ends before compression in order to reduce friction. These two different types of compression tests are denoted hereafter as 'Cylinder' and 'Teflon', respectively. The Rastegaev test specimens where lubricated with Molykote DX paste on the top and bottom ends, including the grooves, before compression. This experiment is denoted hereafter as "Rastegaev". Examples of the compression test specimens, before and after compression, are shown in Fig. 2. The teflon sheets are also shown. 


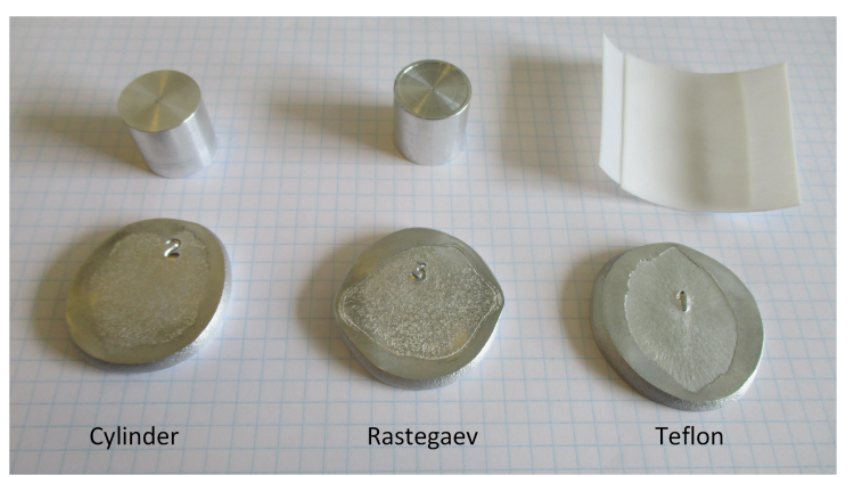

Fig. 2 Compression test specimens before and after deformation

\section{Ring test experiment}

The ring test specimens were machined from the same aluminium rod as the compression test specimens. The dimensions are shown in Fig. 3.

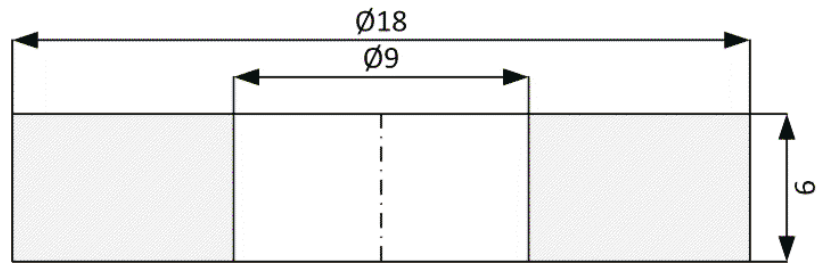

Fig. 3 Geometry of the ring test specimens

Before upsetting, the inner and outer diameter and the height were measured using a Vernier caliper. The rings were lubricated with the same Molykote DX paste or with sheets of teflon that were placed on top and bottom as previously applied in the compression tests. After each upsetting, the smallest inner diameter and final height were measured. Fig. 4 shows the geometry of the rings at different reductions.

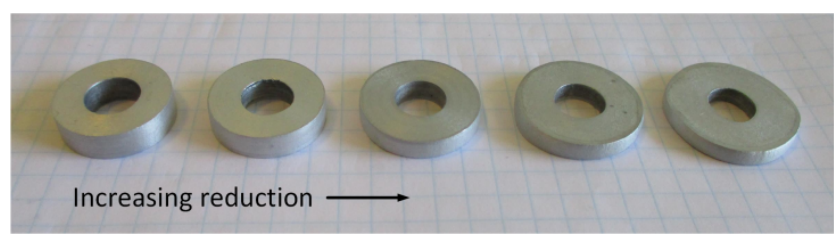

Fig. 4 Rings test specimens after upsetting with Molykote DX paste 
The compression platens used in both compression and ring compression tests had an average roughness $R_{a}=0.2 \mu \mathrm{m}$, but it must be emphasized that surface roughness has no direct relevance for the new proposed methodology to determine the stress-strain curve of metallic materials. In fact, the friction compensation procedure developed by the authors is successful in eliminating all the parameters that are responsible for deviating the evolution of the force with displacement obtained in the real upsetting of cylindrical test specimens from that obtained under homogeneous, frictionless, upsetting of cylindrical test specimens, during which lubrication and surface finish of both specimens and compression platens are supposed to be ideal/perfect.

\section{Results \& Discussion}

\section{Ring test experiments}

The results of the ring test experiments are disclosed in Fig. 5. The calibration curves were obtained with the in-house finite element computer program I-form [18] using a stress-strain curve $\sigma_{o}=$ $131 \bar{\varepsilon}^{0.26}[\mathrm{MPa}]$. Both Coulomb and constant friction models were utilized (refer to Fig. 5a-b).

As it can be seen from Fig. 5a-b, the average Coulomb friction coefficient $\mu_{\text {exp }}=0.12$ and the average friction factor $m_{\text {exp }}=0.23$ when applying DX paste. The standard deviation is 0.0087 and 0.0012 respectively. For the teflon sheets, the average Coulomb friction coefficient is $\mu_{\text {exp }}=0.05$ and the average friction factor $m_{\text {exp }}=0.08$. The standard deviation is 0.0109 and 0.0075 respectively. The average values of the friction coefficient $\left(\mu_{\text {exp }}\right)$ and of the friction factor $\left(m_{\text {exp }}\right)$ where determined from linear interpolation between the lines. 


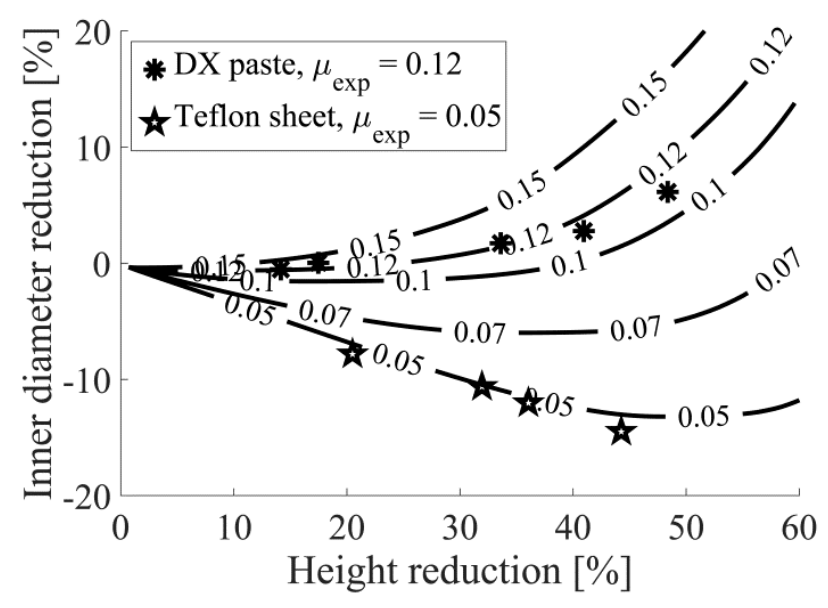

a) Coulomb friction

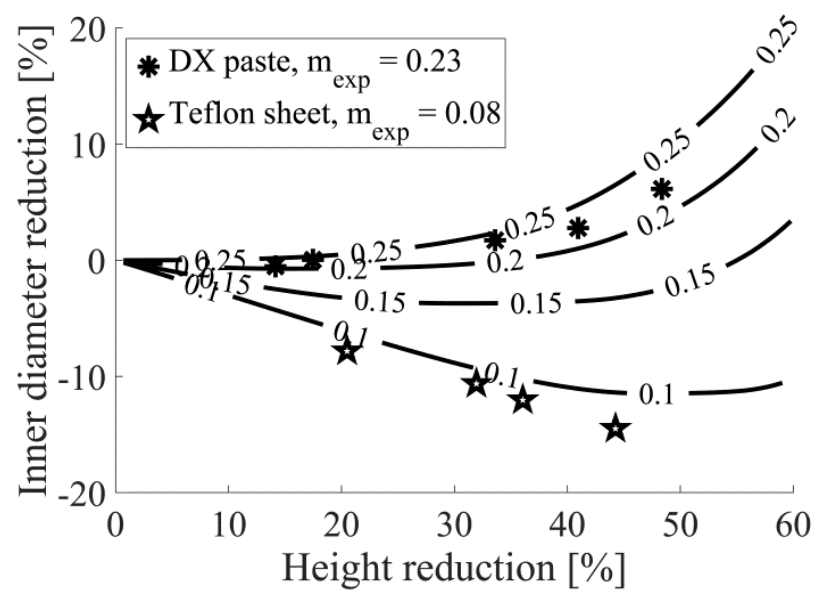

b) Constant friction

Fig. 5 Friction calibration curves with ring experiments

\section{Stress-strain curves}

The determination of the strain hardening material models from the experimental evolution of the force with displacement obtained in cylindrical and Rastegaev compression test specimens, followed the procedure that was previously described in the section entitled 'Theoretical background'. For readability purposes it was decided to include also the results obtained from the first guess of the numerical procedure and to use the Hollomon model. However, the overall numerical procedure can easily be applied to the other strain hardening material models that are listed in Table 1.

\section{Hollomon - frictionless}

The first stage of the proposed method assumes the results of the compression tests to be obtained under homogenous plastic deformation. This allows determining an initial guess for the independent parameters $C$ and $n$ (strength coefficient and strain hardening exponent) of the material strain hardening model (Table 2). The coefficient of determination (R2), to evaluate the quality of the fitted curve to the measurements, is also listed in Table 2 for each test and also listed for the subsequent tests. 
As seen in Table 2 there are differences in the results obtained from the compression tests performed with teflon sheets and from those performed with Molykote DX paste. Differences are also found to the results obtained from the Rastegaev type specimen. The cylinder with Molykote DX paste and the Rastegaev test specimens give approximately similar values of $C$ and $n$ but these are somewhat larger than those obtained for the cylinder with teflon sheets.

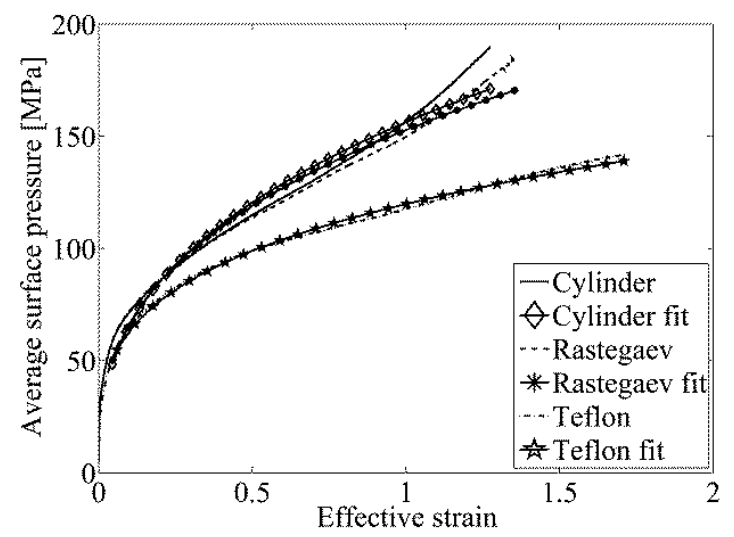

Fig. 6 Compression tests with Hollomon fit

Table 2 Best fit constants based on Hollomon fit

Experiment Strength coefficient C [MPa] Strain hardening exponent $n \quad \mathrm{R}^{2}$

\begin{tabular}{lccc}
\hline Cylinder & 156 & 0.38 & 0.9729 \\
Rastegaev & 153 & 0.36 & 0.9774 \\
Teflon & 120 & 0.28 & 0.9967
\end{tabular}

Fig. 6 shows the evolution of the measured and computed evolution of the average surface pressure with the effective strain, using the material parameters that are included in Table 2. As seen, both the Cylinder and the Rastegaev test specimen present deviations against the 'pure (frictionless)' Hollomon stress-strain behaviour. In contrast, the specimen with teflon sheets presents a fair agreement with the assumed frictionless upsetting of a Hollomon strain hardening material. 


\section{Hollomon - Coulomb friction}

The second stage of the proposed procedure takes friction into account and determines the independent parameters $C$ and $n$ of the material strain hardening model simultaneously with the coefficient of friction $\mu$ (in case of assuming a Coulomb friction model, eq. (2)) The convergence criterion $\delta$ was set equal to $10^{-4}$ and the overall CPU time was less $10 \mathrm{~s}$ until convergence was reached on a laptop computer.

Fig. 7 shows the evolution of the average surface pressure with the effective strain and the constants of the material strain hardening model are given in Table 3.

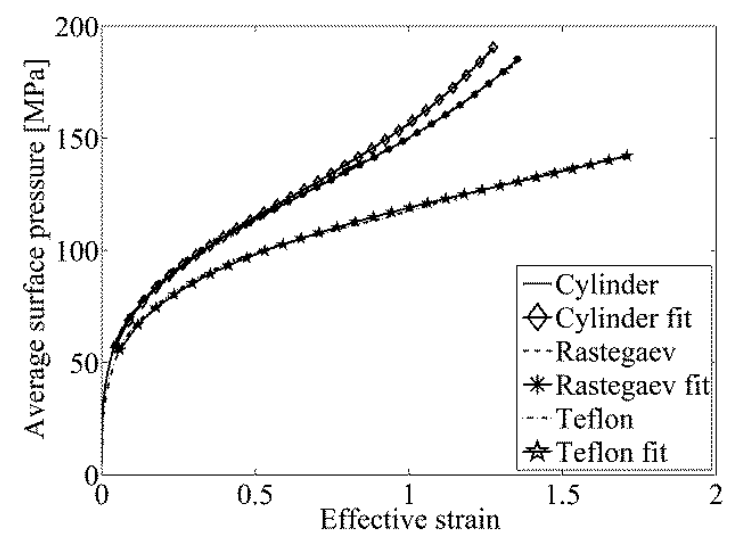

Fig. 7 Compression tests with Hollomon-Coulomb friction fit

Table 3 Best fit constants based on Hollomon-Coulomb friction fit

Experiment Strength coefficient C [MPa] Strain hardening exponent n Friction coefficient $\mu \quad \mathrm{R}^{2}$

\begin{tabular}{lcccc}
\hline Cylinder & 124 & 0.27 & 0.15 & 0.9997 \\
Rastegaev & 126 & 0.27 & 0.11 & 0.9997 \\
Teflon & 117 & 0.26 & 0.01 & 0.9979
\end{tabular}


As can be seen in Fig. 7, there is a good agreement between the final results provided by the proposed model and the experiments. Table 3 also shows that the independent parameters $C$ and $n$ of the material strain hardening model of the Cylinder and Rastegaev experiments are closer to the Teflon test case than in the first guess, when frictionless conditions were assumed. In addition, results also show that the friction coefficient of the Cylinder test $(\mu=0.15)$ is in good agreement with the friction coefficient obtained from the ring tests $\left(\mu_{\text {exp }}=0.12\right)$.

The test performed with the Rastegaev test specimen shows some level of residual friction $(\mu=$ 0.11) in reasonable agreement with the ring test.

The friction coefficient of the compression test performed with teflon sheets $(\mu=0.01)$ is smaller than found by the ring test. This may be due to the rings cutting into the teflon sheets and thereby increasing the friction in the ring test as compared to the compression test.

\section{Hollomon - Constant friction}

Friction is now taken into account based on eq. (3) and corresponding $C$ and $n$ values are computed together with the friction factor $m$. Fig. 9 shows the evolution of the average surface pressure with the effective strain. The constants of the strain hardening material model are included in Table 5.

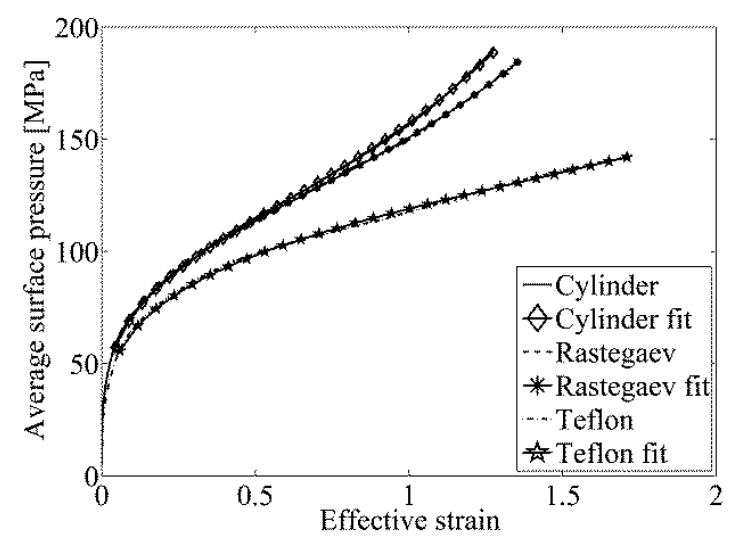

Fig. 8 Compression experiments with Hollomon-Constant friction fit 
Table 4 Best fit constants based on Hollomon-Constant friction fit

Experiment Strength coefficient C $[\mathrm{MPa}] \quad$ Strain hardening exponent $\mathrm{n} \quad$ Friction factor $\mathrm{m} \quad \mathrm{R}^{2}$

\begin{tabular}{lcccc}
\hline Cylinder & 118 & 0.26 & 0.38 & 0.9996 \\
Rastegaev & 123 & 0.26 & 0.27 & 0.9997 \\
Teflon & 117 & 0.26 & 0.02 & 0.9979
\end{tabular}

Fig. 8 shows a good agreement between eq. (3) and the experiments. Table 4 shows a reasonable agreement between the three strength coefficients and the strain hardening exponents. However the friction factor for the Cylinder experiment $(m=0.38)$ is too large when compared with that obtained from ring tests $\left(m_{\text {exp }}=0.23\right)$.

In contrast, the Rastegaev experiment shows a friction factor similar to the one obtained in the ring tests. This result is easy to understand because the contact geometry of both tests (along a ring zone) is somewhat identical.

The Teflon experiment presents a small friction factor and is once again very close to frictionless conditions and smaller than found by the ring test.

\section{Hollomon - Combined friction}

Friction is now taken into account based on eq. (5). The $C$ and $n$ values are computed together with the friction coefficient $\mu$, the friction factor $m$ and the radius ratio $x=r_{g} / R$, according to the objective function $f\left(M, \tau_{s}, x\right)$. Fig. 9 provides the evolution of the average surface pressure with the effective strain and the material strain hardening independent parameters are given in Table 5. 


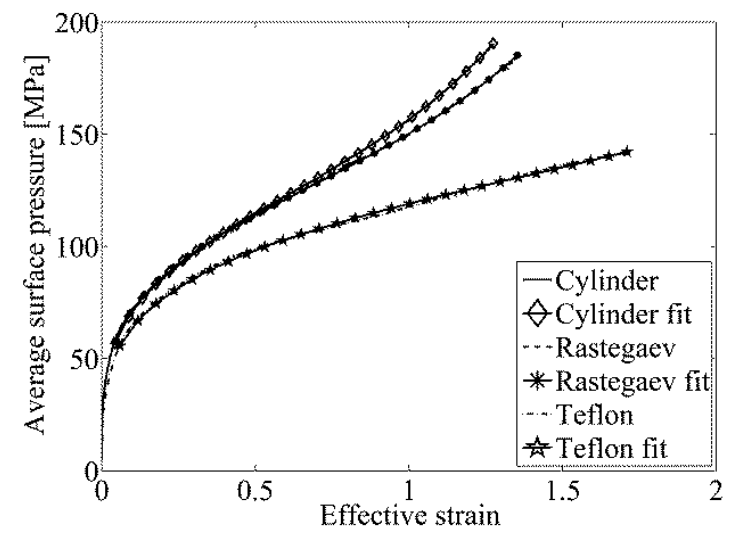

Fig. 9 Compression experiments with Hollomon-combined friction fit

Table 5 Best fit constants based on Hollomon-Mixed friction fit Experiment Strength coef. C $[\mathrm{MPa}] \quad$ Strain hard. exp. $\mathrm{n} \quad$ Fric. factor $\mathrm{m} \quad$ Fric. coef. $\mu \quad$ Radius ratio $\mathrm{x} \quad \mathrm{R}^{2}$

\begin{tabular}{lcccccc}
\hline Cylinder & 124 & 0.27 & 0.21 & 0.15 & 0.10 & 0.9997 \\
Rastegaev & 127 & 0.27 & 0.10 & 0.11 & 0.08 & 0.9997 \\
Teflon & 117 & 0.26 & 0.02 & 0.01 & 0.00 & 0.9979
\end{tabular}

Results show a reasonable agreement between eq. (5) and the experiments. Table 5 indicates a fair agreement between the strength coefficient $C$ and the strain hardening exponent $n$ of the three experiments. In case of the Cylinder, there is also a good agreement between the calculated and experimental values of the friction factor and the friction coefficient $\left(\mu_{\text {exp }}=0.12\right.$ and $m_{\exp }=$ 0.23). It can also be seen that although the radius ratio $x$ is fairly small for all the experiments it is large enough to justify the need for using a combined friction model in case of the Cylinder and the Rastegaev specimens. This could explain why the Cylinder experiment predicts a somewhat larger value of the friction factor, when applying eq. (3), than that found by means of the ring test experiment. 
In conclusion it may also be said that frictional conditions are better modelled by the Coulomb friction model in case of compression tests performed for the experimental conditions that are used in this investigation.

\section{Conclusion}

A numerical procedure has been proposed which enables to compensate for friction when performing upsetting compression tests. The procedure has been tested on two different geometries; Cylinder and Rastegaev. The cylinders were lubricated with either grease or teflon sheets on top and bottom ends while the Rastegaev’s specimens were lubricated with grease.

When the stress-strain curve is directly obtained from the experimentally measured loaddisplacement curves, there are differences resulting from the type of specimen and associated lubrication procedure. However, these differences disappear when the effect of friction on the compression load is corrected by means of the proposed methodology and similar values of strength coefficient and of the strain hardening exponent are obtained.

The application of a combined friction model resulting from combination of the Coulomb and constant friction laws in the determination of the stress-strain curve is considered to provide the best results because the friction coefficient and the friction factor of the upset compression tests are similar to those obtained by means of independent ring compression tests.

\section{Acknowledgment}

The authors would like to acknowledge the support provided by The Danish Council for Independent Research under the grant number DFF - 4005-00130. Paulo Martins would also like to acknowledge the support provided by Fundação para a Ciência e a Tecnologia of Portugal under LAETA - UID/EMS/50022/2013 and PTDC/EMS-TEC/0626/2014. 


\section{References}

1 Rastegaev MV (1940) Neue Methode der homogenen Stauchung, Zavodskaja Laboratoria 354-355

2 Cook M, Larke EC (1945) Resistance of copper and copper alloys to homogeneous deformation in compression. J. Inst. Metals 71: 371-390

3 Alexander JM, Brewer RC (1963) Manufacturing Properties of Materials, Van Nostrand, London

4 Watts AB, Ford H (1955) On the basic yield stress curve for a metal. P I MECH ENG 169: 1141-1156

5 Woodward RL (1977) A note on the determination of accurate flow properties from simple compression tests. Metallurgical Transactions A 8A: 1833-1834

6 Avitzur B (1968) Metal forming: Processes and Analysis. McGraw Hill, New York

7 Osakada K, Kawasaki T, Mori K (1981) A Method of Determining Flow Stress under Forming Conditions. Annals of CIRP 30: 135-138

8 Han Han (2002) The validity of the mathematical models evaluated by two-specimen method under the unknown coefficient of friction and flow stress. J. Mater. Process. Technol. 122: 386-396

$9 \quad$ Mielnik E (1991) Metalworking Science and Engineering McGraw Hill, New York

10 Xinbo L, Fubao Z, Zhiliang Z (2002) Determination of metal material flow stress by the method of C-FEM. J. Mater. Process. Technol. 120: 144-150

11 Tan X, Zhang W, Bay N (1999) A new friction test using simple upsetting and flow analysis. Advanced Technology of Plasticity, 1: 365-370

12 Wilson WRD (1979) Friction and Lubrication in Bulk Metal-Forming Processes. J. Applied Metalworking 1:1-19 
13 Hollomon JH (1945) Tensile Deformation. T Am I Min Met Eng 162:268-290

14 Swift HW (1952) Plastic instability under plane strain. J Mechan Phys Solids 1:1-18

15 Ludwik P (1909) Elemente der Technologischen Mechanik. Springer Verlag, Berlin

16 Voce E (1948) The relationship between stress and strain for homogeneous deformation. J Inst Metals 74:537-562

17 Chapra S, Canale R (2009) Numerical Methods for Engineers. McGraw-Hill

18 Nielsen CV, Zhang W, Alves LM, Bay N, Martins PAF (2013) Modelling of Thermo-ElectroMechanical Manufacturing Processes with Applications in Metal Forming and Resistance Welding. Springer-Verlag, London 


\section{Appendix 1}

A pseudo-code is listed in the appendix enabling the reader to perform friction correction in upsetting experiments. The used names for variables are explained during the code. The pseudo-code is written in MATLAB version 14.

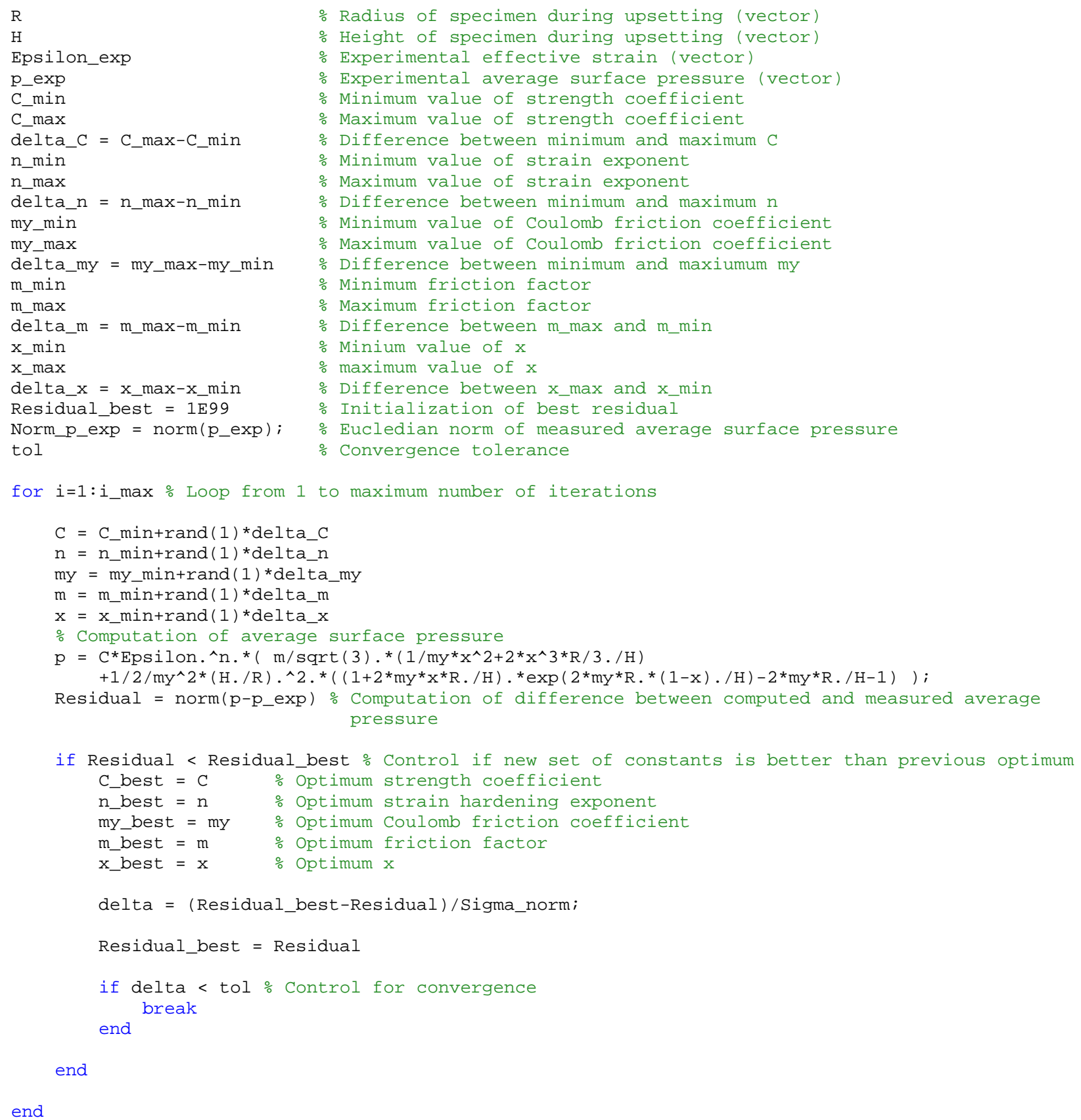

